\title{
Long range correlations and phase transition in non-equilibrium diffusive systems
}

\author{
T. Bodineau, B. Derrida ${ }^{\dagger}$, V. Lecomte ${ }^{\ddagger}$ and F. van Wijland ${ }^{\S}$
}

October 30, 2018

\begin{abstract}
We obtain explicit expressions for the long range correlations in the $\mathrm{ABC}$ model and in diffusive models conditioned to produce an atypical current of particles. In both cases, the two-point correlation functions allow to detect the occurrence of a phase transition as they become singular when the system approaches the transition.
\end{abstract}

\section{Introduction}

A generic property of non-equilibrium systems, maintained in a steady state by contact with several reservoirs of particles at unequal chemical potentials (or by contact with several heat baths at unequal temperatures), is the presence of long range correlations [1, 2, 3, 4, 5, 6]. In diffusive systems, these long range correlations and their dependence on the system size [1, 17] can be related to the non-local nature of the large deviation function of the density [8]. Another generic property of non-equilibrium systems is the possibility of phase transitions for one dimensional systems with short range interactions

*Ecole Normale Supérieure, DMA, 45 rue d'Ulm 75230 Paris cedex 05, France

${ }^{\dagger}$ Laboratoire de Physique Statistique (CNRS UMR 8550), École Normale Supérieure, 24 rue Lhomond, 75231 Paris cedex 05, France

†Département de Physique de la Matière Condensée, Université de Genève, 24 quai Ernest Ansermet, 1211 Genève, Switzerland

$\S$ Laboratoire Matière et Systèmes Complexes (CNRS UMR 7057), 10 rue Alice Domon et Léonie Duquet, Université Paris Diderot, 75205 Paris cedex 13, France 
[9, 10, 11, 12, 13, 14, 15, 16, 18, 19, 20, in contrast with equilibrium systems where such phase transitions are excluded by the well known Landau argument.

The goal of the present paper is to calculate these long range correlations, for two examples of diffusive systems, and to show that they become singular at the phase transition, even when the analytic expression of quantities such as the average current or its large deviation function has no singularity as one approaches the phase transition.

The paper is organized as follows: in section 2, we analyze the ABC model [12, 13, 14, 15, 18, for which we compute first the correlation functions in the case of equal densities of the three species, from the known large deviation function of the density. We then obtain the correlation functions in the general case, i.e. for arbitrary densities, by two methods: a simple truncation procedure based on the expected scaling on the system size of the various correlation functions and the macroscopic fluctuation theory which was used recently by Bertini, De Sole, Gabrielli, Jona-Lasinio, Landim [21, 22, 23, 24] in the context of non-equilibrium systems.

In section 3 we consider the weakly asymmetric exclusion process, for which a phase transition is expected to occur, in the large deviation function of the current. We calculate the pair correlation functions, conditioned on a current deviation, and show that they become singular at the transition.

\section{The ABC model}

The ABC model [12, 13, 14, 15, 18] describes a system of three species $A, B, C$ of particles on a one dimensional ring of $L$ lattice sites. Each site is occupied by one and only one of these three types of particles. The dynamics are fully specified by the following exchange rates between neighboring sites:

$$
\begin{aligned}
& A B \underset{1}{\stackrel{q}{\rightleftarrows}} B A \\
& B C \underset{1}{\stackrel{q}{\rightleftarrows}} C B \\
& C A \underset{1}{\stackrel{q}{\rightleftarrows}} A C
\end{aligned}
$$

where $q \leq 1$ (the range $q>1$ is related to the case $q<1$ by a left-right symmetry). Since the number of particles of each species is conserved by the dynamics, the properties of the system depend on the global densities 
$r_{A}, r_{B}, r_{C}$ of the three species and on the parameter $q$. As all sites are occupied by one of the three species, one has of course

$$
r_{A}+r_{B}+r_{C}=1
$$

When $q=1$ all allowed configurations are equally likely (and the steady state density profiles of the three species are flat) but as $q$ decreases, the different species tend to segregate and in the limit $q \rightarrow 0$ the steady state consists of three clusters: a cluster of all $A$ 's followed by a cluster of all $B$ 's which is itself followed by a cluster of all $C$ 's.

Let us first briefly recall some known properties of the ABC model [14]. As $q$ varies, with the following scaling depending on the size $L$ of the ring,

$$
q=\exp \left[-\frac{\beta}{L}\right]
$$

one observes (for large $L$ ) phase transitions, in the steady state, at some critical value $\beta_{c}$.

For equal densities $\left(r_{A}=r_{B}=r_{C}=\frac{1}{3}\right)$, it is known that

$$
\beta_{c}=2 \pi \sqrt{3}
$$

and that for $\beta<\beta_{c}$ the density profiles of the three species are flat, while they become space-dependent for $\beta>\beta_{c}$.

A stability analysis of the flat phase leads to the following prediction of $\beta_{c}$ in the general case

$$
\beta_{c}=\frac{2 \pi}{\left[1-2\left(r_{A}^{2}+r_{B}^{2}+r_{C}^{2}\right)\right]^{1 / 2}}
$$

which should be the exact expression as long as the transition remains a second order phase transition. One expects, however, a first order phase transition to occur for some range of densities at least when $r_{A}^{2}+r_{B}^{2}+r_{C}^{2}<$ $2\left(r_{A}^{3}+r_{B}^{3}+r_{C}^{3}\right)$, and the precise location of this first order transition is not known. In the flat phase, the steady state currents have expressions

$$
J_{A}=\beta \frac{r_{A}\left(r_{C}-r_{B}\right)}{L}
$$

(and similar expressions for $J_{B}$ and $J_{C}$ ) which do not show any singularity as $\beta \rightarrow \beta_{c}^{-}$. 
In the steady state, the probability of observing macroscopic profiles $\rho_{A}(x), \rho_{B}(x), \rho_{C}(x)$ (where $0 \leq x<1$ is a macroscopic coordinate along the ring) has the following large $L$ dependence

$$
\operatorname{Pro}\left(\rho_{A}(x), \rho_{B}(x), \rho_{C}(x)\right) \sim \exp \left(-L \mathcal{F}\left[\rho_{A}(x), \rho_{B}(x), \rho_{C}(x)\right]\right) .
$$

The large deviation function $\mathcal{F}$ of the density profiles is known for the $\mathrm{ABC}$ model when $r_{A}=r_{B}=r_{C}=\frac{1}{3}$ :

$$
\begin{aligned}
& \mathcal{F}\left[\rho_{A}(x), \rho_{B}(x), \rho_{C}(x)\right]= \\
& \quad \kappa+\int_{0}^{1} d x\left[\rho_{A}(x) \ln \rho_{A}(x)+\rho_{B}(x) \ln \rho_{B}(x)+\rho_{C}(x) \ln \rho_{C}(x)\right] \\
& \quad+\beta \int_{0}^{1} d x \int_{0}^{1} d z z\left[\rho_{B}(x) \rho_{C}(x+z)+\rho_{C}(x) \rho_{A}(x+z)+\rho_{A}(x) \rho_{B}(x+z)\right] .
\end{aligned}
$$

where $\kappa$ is a normalisation constant but it is not known for arbitrary $r_{A}, r_{B}, r_{C}$. So far it has only been computed in [14] to order $\beta^{2}$

$$
\begin{aligned}
& \mathcal{F}\left[\rho_{A}(x), \rho_{B}(x), \rho_{C}(x)\right]= \\
& \kappa+\int_{0}^{1} d x\left[\rho_{A}(x) \ln \rho_{A}(x)+\rho_{B}(x) \ln \rho_{B}(x)+\rho_{C}(x) \ln \rho_{C}(x)\right] \\
& +\beta \int_{0}^{1} d x \int_{0}^{1} d z z\left[\rho_{B}(x) \rho_{C}(x+z)+\rho_{C}(x) \rho_{A}(x+z)+\rho_{A}(x) \rho_{B}(x+z)\right] \\
& -\frac{3}{4} \beta^{2} \int_{0}^{1} d x \int_{0}^{1} d z z(1-z)\left[r_{A}\left(1-3 r_{A}\right) \rho_{B}(x) \rho_{C}(x+z)\right. \\
& \left.\quad+r_{B}\left(1-3 r_{B}\right) \rho_{C}(x) \rho_{A}(x+z)+r_{C}\left(1-3 r_{C}\right) \rho_{A}(x) \rho_{B}(x+z)\right] \\
& \quad+O\left(\beta^{3}\right)
\end{aligned}
$$

\subsection{The correlation function in the equal density case}

When the large deviation function is known, as in (7) for the equal density case, the calculation of the correlation functions in the flat phase can be easily done from the contribution of the fluctuations of density around the flat profiles. For a small perturbation of the form

$$
\begin{aligned}
& \rho_{A}(x)=\frac{1}{3}+a_{1} \cos (2 \pi n x)+a_{2} \sin (2 \pi n x) \\
& \rho_{B}(x)=\frac{1}{3}+b_{1} \cos (2 \pi n x)+b_{2} \sin (2 \pi n x)
\end{aligned}
$$


with $n \geq 1$ and $\rho_{C}(x)=1-\rho_{A}(x)-\rho_{B}(x)$, one gets from (7) at quadratic order in $a_{1}, a_{2}, b_{1}, b_{2}$

$$
\begin{aligned}
& \operatorname{Pro}\left(\rho_{A}(x), \rho_{B}(x), \rho_{C}(x)\right) \sim \\
& \quad \exp \left[-\frac{3 L}{2}\left(a_{1}^{2}+a_{1} b_{1}+b_{1}^{2}+a_{2}^{2}+a_{2} b_{2}+b_{2}^{2}\right)+\frac{3 L \beta}{4 n \pi}\left(a_{1} b_{2}-a_{2} b_{1}\right)\right]
\end{aligned}
$$

Therefore, the covariances in the steady state of the $n$-th Fourier coefficients of the densities $\rho_{A}(x)$ and $\rho_{B}(x)$ are

$$
\begin{gathered}
\left\langle a_{1}^{2}\right\rangle=\left\langle a_{2}^{2}\right\rangle=\left\langle b_{1}^{2}\right\rangle=\left\langle b_{2}^{2}\right\rangle=\frac{16 n^{2} \pi^{2}}{3 L\left(12 n^{2} \pi^{2}-\beta^{2}\right)} \\
\left\langle a_{1} a_{2}\right\rangle=\left\langle b_{1} b_{2}\right\rangle=0 \\
\left\langle a_{1} b_{1}\right\rangle=\left\langle a_{2} b_{2}\right\rangle=-\frac{8 n^{2} \pi^{2}}{3 L\left(12 n^{2} \pi^{2}-\beta^{2}\right)} \\
\left\langle a_{1} b_{2}\right\rangle=-\left\langle a_{2} b_{1}\right\rangle=\frac{4 \pi n \beta}{3 L\left(12 n^{2} \pi^{2}-\beta^{2}\right)}
\end{gathered}
$$

Then if one sums over all the fluctuations, i.e. over all the wave numbers $n$ on the ring, one obtains

$$
\begin{gathered}
\left\langle\rho_{A}(x) \rho_{A}(y)\right\rangle_{c}=\frac{16 \pi^{2}}{3 L} \sum_{n \geq 1} \frac{n^{2} \cos (2 \pi n(y-x))}{\left(12 n^{2} \pi^{2}-\beta^{2}\right)} \\
\left\langle\rho_{A}(x) \rho_{B}(y)\right\rangle_{c}=\frac{-8 \pi^{2}}{3 L} \sum_{n \geq 1} \frac{n^{2} \cos (2 \pi n(y-x))}{\left(12 n^{2} \pi^{2}-\beta^{2}\right)}+\frac{4 \pi \beta}{3 L} \sum_{n \geq 1} \frac{n \sin (2 \pi n(y-x))}{\left(12 n^{2} \pi^{2}-\beta^{2}\right)}(10)
\end{gathered}
$$

and similar expressions for the other correlation functions (we will see below in (36]37) a different way of writing these expressions). Clearly these expressions become singular as $\beta$ approaches the critical value $\beta_{c}=2 \pi \sqrt{3}$.

\subsection{The correlation function for arbitrary densities by a simple truncation procedure}

Let us introduce the notation $A_{i}=1$ when site $i$ is occupied by a $A$ particle, $A_{i}=0$ otherwise, and similarly $B_{i}$ or $C_{i}$ for a $B$ or a $C$ particle, so that on each site $i$ one has

$$
A_{i}+B_{i}+C_{i}=1
$$


One can derive from the dynamical rule (1) the following exact evolution equations:

$$
\begin{aligned}
\frac{d\left\langle A_{i}\right\rangle}{d t} & =q\left\langle A_{i-1} B_{i}\right\rangle+\left\langle B_{i} A_{i+1}\right\rangle-q\left\langle A_{i} B_{i+1}\right\rangle-\left\langle B_{i-1} A_{i}\right\rangle \\
& +\left\langle A_{i-1} C_{i}\right\rangle+q\left\langle C_{i} A_{i+1}\right\rangle-\left\langle A_{i} C_{i+1}\right\rangle-q\left\langle C_{i-1} A_{i}\right\rangle
\end{aligned}
$$

where \langle\rangle stands for the expectation with respect to the dynamics. For $|j-i| \geq 2$

$$
\begin{aligned}
\frac{d\left\langle A_{i} A_{j}\right\rangle}{d t}= & q\left\langle A_{i-1} B_{i} A_{j}\right\rangle+\left\langle B_{i} A_{i+1} A_{j}\right\rangle-q\left\langle A_{i} B_{i+1} A_{j}\right\rangle-\left\langle B_{i-1} A_{i} A_{j}\right\rangle \\
+ & q\left\langle A_{i} A_{j-1} B_{j}\right\rangle+\left\langle A_{i} B_{j} A_{j+1}\right\rangle-q\left\langle A_{i} A_{j} B_{j+1}\right\rangle-\left\langle A_{i} B_{j-1} A_{j}\right\rangle \\
& +\left\langle A_{i-1} C_{i} A_{j}\right\rangle+q\left\langle C_{i} A_{i+1} A_{j}\right\rangle-\left\langle A_{i} C_{i+1} A_{j}\right\rangle-q\left\langle C_{i-1} A_{i} A_{j}\right\rangle \\
& +\left\langle A_{i} A_{j-1} C_{j}\right\rangle+q\left\langle A_{i} C_{j} A_{j+1}\right\rangle-\left\langle A_{i} A_{j} C_{j+1}\right\rangle-q\left\langle A_{i} C_{j-1} A_{j}\right\rangle \\
\frac{d\left\langle A_{i} B_{j}\right\rangle}{d t}= & q\left\langle A_{i-1} B_{i} B_{j}\right\rangle+\left\langle B_{i} A_{i+1} B_{j}\right\rangle-q\left\langle A_{i} B_{i+1} B_{j}\right\rangle-\left\langle B_{i-1} A_{i} B_{j}\right\rangle \\
& +q\left\langle A_{i} B_{j-1} C_{j}\right\rangle+\left\langle A_{i} C_{j} B_{j+1}\right\rangle-q\left\langle A_{i} B_{j} C_{j+1}\right\rangle-\left\langle A_{i} C_{j-1} B_{j}\right\rangle \\
& +\left\langle A_{i-1} C_{i} B_{j}\right\rangle+q\left\langle C_{i} A_{i+1} B_{j}\right\rangle-\left\langle A_{i} C_{i+1} B_{j}\right\rangle-q\left\langle C_{i-1} A_{i} B_{j}\right\rangle \\
& +\left\langle A_{i} B_{j-1} A_{j}\right\rangle+q\left\langle A_{i} A_{j} B_{j+1}\right\rangle-\left\langle A_{i} B_{j} A_{j+1}\right\rangle-q\left\langle A_{i} A_{j-1} B_{j}\right\rangle
\end{aligned}
$$

and for $j=i+1$ :

$$
\begin{array}{r}
\frac{d\left\langle A_{i} B_{i+1}\right\rangle}{d t}=q\left\langle A_{i-1} B_{i} B_{i+1}\right\rangle+\left\langle B_{i} A_{i+1}\right\rangle-q\left\langle A_{i} B_{i+1}\right\rangle-\left\langle B_{i-1} A_{i} B_{i+1}\right\rangle \\
+\left\langle A_{i} C_{i+1} B_{i+2}\right\rangle-q\left\langle A_{i} B_{i+1} C_{i+2}\right\rangle \\
+\left\langle A_{i-1} C_{i} B_{i+1}\right\rangle-q\left\langle C_{i-1} A_{i} B_{i+1}\right\rangle+q\left\langle A_{i} A_{i+1} B_{i+2}\right\rangle-\left\langle A_{i} B_{i+1} A_{i+2}\right\rangle
\end{array}
$$

and similar expressions for the evolution of the other one or two point functions. In the steady state, the left hand sides of the equations (12) vanish.

For diffusive systems, one expects the long range part of the $p$-point connected part of the correlation functions to scale as $L^{1-p}$ in the steady state [1, 7, 8]. This means that, for large $L$, in the flat phase of the $A B C$ model,

$$
\left\langle A_{i} B_{j}\right\rangle=\left\langle A_{i}\right\rangle\left\langle B_{j}\right\rangle+\left\langle A_{i} B_{j}\right\rangle_{c}=r_{A} r_{B}+\frac{1}{L} F_{a b}\left(\frac{j-i}{L}\right)
$$


where $F_{a b}$ stands for the normalized connected two-point function and

$$
\begin{aligned}
\left\langle A_{i} B_{j} C_{k}\right\rangle=r_{A} r_{B} r_{C} & +\frac{1}{L}\left[r_{C} F_{a b}\left(\frac{j-i}{L}\right)+r_{B} F_{a c}\left(\frac{k-i}{L}\right)+r_{A} F_{b c}\left(\frac{k-j}{L}\right)\right] \\
& +\frac{1}{L^{2}} G_{a b c}\left(\frac{j-i}{L}, \frac{k-i}{L}\right)
\end{aligned}
$$

where $G_{a b c}$ stands for the normalized connected three-point function. Using similar notations for all the other 2-point and 3-point functions and the relation (11), and replacing the various correlation functions in (13,14,15) by their scaling forms (16]17), one gets to leading orders in $L^{-1}$ (order $L^{-3}$ for (13) 14), and order $L^{-1}$ for (15))

$$
\begin{aligned}
& F_{a a}(x)^{\prime \prime}=\beta r_{A}\left[F_{b a}(x)^{\prime}-F_{a b}(x)^{\prime}\right] \\
& F_{b b}(x)^{\prime \prime}=\beta r_{B}\left[F_{b a}(x)^{\prime}-F_{a b}(x)^{\prime}\right] \\
& F_{a b}(x)^{\prime \prime}=\beta\left[r_{B} F_{a a}(x)^{\prime}+r_{A} F_{b b}(x)^{\prime}-\left(1-2 r_{A}-2 r_{B}\right) F_{a b}(x)^{\prime}\right] \\
& F_{b a}(x)^{\prime \prime}=\beta\left[-r_{B} F_{a a}(x)^{\prime}-r_{A} F_{b b}(x)^{\prime}+\left(1-2 r_{A}-2 r_{B}\right) F_{b a}(x)^{\prime}\right]
\end{aligned}
$$

as well as

$$
F_{a b}(0)-F_{b a}(0)=3 \beta r_{A} r_{B} r_{C} .
$$

Note that in (18) at leading order in $1 / L$, the contribution of the functions G's which appear in (17) drops out. Note also that

$$
F_{a b}(x)=F_{b a}(1-x) .
$$

The general solution of (18) is

$$
\begin{gathered}
F_{a a}(x)=K_{a a}-2 K \beta r_{A} \cos \left(\frac{\beta \sqrt{\Delta}}{2}(2 x-1)\right) \\
F_{b b}(x)=K_{b b}-2 K \beta r_{B} \cos \left(\frac{\beta \sqrt{\Delta}}{2}(2 x-1)\right) \\
F_{a b}(x)=F_{b a}(1-x)=K_{a b}+K\left(1-2 r_{C}\right) \beta \cos \left(\frac{\beta \sqrt{\Delta}}{2}(2 x-1)\right) \\
-K \beta \sqrt{\Delta} \sin \left(\frac{\beta \sqrt{\Delta}}{2}(2 x-1)\right)
\end{gathered}
$$

where $K, K_{a a}, K_{a b}, K_{b b}$ are integration constants and

$$
\Delta=1-2\left(r_{A}^{2}+r_{B}^{2}+r_{C}^{2}\right) .
$$


Relation (19) leads to

$$
K=-\frac{3 r_{A} r_{B} r_{C}}{2 \sqrt{\Delta} \sin \frac{\beta \sqrt{\Delta}}{2}}
$$

The remaining constants can be determined by using the fact that

$$
\sum_{k=1}^{L-1}\left\langle A_{i} A_{i+k}\right\rangle=\frac{N_{A}\left(N_{A}-1\right)}{L}
$$

and similar sum rules for the other correlation functions which imply that

$\int_{0}^{1} F_{a a}(x) d x=-r_{A}\left(1-r_{A}\right), \int_{0}^{1} F_{b b}(x) d x=-r_{B}\left(1-r_{B}\right), \int_{0}^{1} F_{a b}(x) d x=r_{A} r_{B}$

and the final result is for $0<x<1$

$F_{a a}(x)=-r_{A}\left(1-r_{A}\right)-\frac{3 r_{A}^{2} r_{B} r_{C}}{\sqrt{\Delta} \sin \frac{\beta \sqrt{\Delta}}{2}}\left(\beta \cos \left(\frac{\beta \sqrt{\Delta}}{2}(2 x-1)\right)-2 \frac{\sin \left(\frac{\beta \sqrt{\Delta}}{2}\right)}{\sqrt{\Delta}}\right)$

$$
\begin{array}{r}
F_{a b}(x)=r_{A} r_{B}+\frac{3 r_{A} r_{B} r_{C}}{2 \sqrt{\Delta} \sin \frac{\beta \sqrt{\Delta}}{2}}\left[\left(1-2 r_{C}\right)\left(\beta \cos \left(\frac{\beta \sqrt{\Delta}}{2}(2 x-1)\right)-2 \frac{\sin \left(\frac{\beta \sqrt{\Delta}}{2}\right)}{\sqrt{\Delta}}\right)\right. \\
\left.-\beta \sqrt{\Delta} \sin \left(\frac{\beta \sqrt{\Delta}}{2}(2 x-1)\right)\right]
\end{array}
$$

As $\beta \rightarrow \frac{2 \pi}{\sqrt{\Delta}}$ the correlation functions diverge and one recovers the expression (5) of the second order transition.

As starting from (16]17) we only considered the correlations in the flat phase, our truncation procedure is unable to distinguish between a stable and a metastable phase and to predict the occurrence of first order transitions. Thus we cannot exclude that the flat phase becomes metastable for some values of $\beta$ smaller than $\beta_{c}$ given by (5).

\subsection{The macroscopic fluctuation theory}

In the $A B C$ model there is always one and only one particle per site. One can then describe the system on a macroscopic scale and on a diffusive time, i.e. on times which scale as $L^{2}$, by only two density profiles $\rho_{A}(x, \tau), \rho_{B}(x, \tau)$. 
The key quantities to the macroscopic description of the system are the typical currents for given density profiles and their variance in the steady state. Let $Q_{t}^{A}(i), Q_{t}^{B}(i)$ be the fluxes of $A, B$ particles between sites $i$ and $i+1$ during a long microscopic time $t$. Starting at time 0 from smooth macroscopic profiles $\rho_{A}(x), \rho_{B}(x)$, the densities evolve according to [14]

$$
\begin{aligned}
& \partial_{\tau} \rho_{A}(x, \tau)=\partial_{x}^{2} \rho_{A}(x, \tau)+\beta \partial_{x}\left(\rho_{A}(x, \tau)\left(\rho_{B}(x, \tau)-\rho_{C}(x, \tau)\right)\right) \\
& \partial_{\tau} \rho_{B}(x, \tau)=\partial_{x}^{2} \rho_{B}(x, \tau)+\beta \partial_{x}\left(\rho_{B}(x, \tau)\left(\rho_{C}(x, \tau)-\rho_{A}(x, \tau)\right)\right)
\end{aligned}
$$

where the macroscopic time $\tau$ scales like $t / L^{2}$. One can understand (25) from (12) by saying that for large $L$, there is a local equilibrium at position $x$, characterized by the densities $\rho_{A}(x, \tau)$ and $\rho_{B}(x, \tau)$. In this local equilibrium, the hydrodynamic currents are given by

$$
\begin{aligned}
& q_{A}=-\frac{d \rho_{A}}{d x}-\beta \rho_{A}\left(\rho_{B}-\rho_{C}\right) \\
& q_{B}=-\frac{d \rho_{B}}{d x}-\beta \rho_{B}\left(\rho_{C}-\rho_{A}\right)
\end{aligned}
$$

To compute the covariance matrix of the currents, it is enough to consider the system in equilibrium with $\beta=0$ and constant densities $\rho_{A}, \rho_{B}$. In this case, the $A$-particles evolve as a Symmetric Simple Exclusion Process (SSEP) (the $B, C$ particles play the role of holes) and the variance of the total current $Q_{t}^{A}=\sum_{i=1}^{L} Q_{t}^{A}(i)$ is given by [17]

$$
\sigma_{A A}=\lim _{t \rightarrow \infty} \frac{1}{t L}\left\langle\left(Q_{t}^{A}\right)^{2}\right\rangle=2 \rho_{A}\left(1-\rho_{A}\right) .
$$

In the same way, the variance of $Q_{t}^{B}=\sum_{i=1}^{L} Q_{t}^{B}(i)$ is given by $\sigma_{B B}=2 \rho_{B}(1-$ $\left.\rho_{B}\right)$. Finally, if we view the $C$-particles as holes and the species $A, B$ as a single type of particle, then the evolution is the same as the SSEP at density $\rho_{A}+\rho_{B}$, so that

$\lim _{t \rightarrow \infty} \frac{1}{t L}\left\langle\left(Q_{t}^{A}+Q_{t}^{B}\right)^{2}\right\rangle=2\left(\rho_{A}+\rho_{B}\right)\left(1-\rho_{A}-\rho_{B}\right)=\sigma_{A A}+\sigma_{B A}+2 \sigma_{A B}$.

where the covariance between the currents of $A$ and $B$ is then $\sigma_{A B}=-2 \rho_{A} \rho_{B}$.

One can adapt the macroscopic fluctuation theory [31, 32] to predict that the probability of observing density profiles $\rho_{A}(x, \tau), \rho_{B}(x, \tau)$ and (rescaled) 
currents $j_{A}(x, \tau), j_{B}(x, \tau)$ over a (rescaled) time interval $0<\tau<T$ is given (this rescaling is discussed for example in section 5 of [28])

$$
\operatorname{Pro}\left(\rho_{A}, \rho_{B}, j_{A}, j_{B}\right) \sim \exp \left(-L \mathcal{I}_{[0, T]}\left(\rho_{A}, \rho_{B}, j_{A}, j_{B}\right)\right)
$$

where

$$
\mathcal{I}_{[0, T]}\left(\rho_{A}, \rho_{B}, j_{A}, j_{B}\right)=\int_{0}^{1} d x \int_{0}^{T} d \tau \mathcal{H}\left(\rho_{A}, \rho_{B}, j_{A}, j_{B}\right)
$$

with

$$
\begin{array}{r}
\mathcal{H}\left(\rho_{A}, \rho_{B}, j_{A}, j_{B}\right)=\frac{1}{2}\left(\begin{array}{c}
j_{A}-q_{A} \\
j_{B}-q_{B}
\end{array}\right)\left(\begin{array}{cc}
\sigma_{A A} & \sigma_{A B} \\
\sigma_{A B} & \sigma_{B B}
\end{array}\right)^{-1}\left(\begin{array}{c}
j_{A}-q_{A} \\
j_{B}-q_{B}
\end{array}\right)(29) \\
=\frac{\sigma_{B B}\left(j_{A}-q_{A}\right)^{2}-2 \sigma_{A B}\left(j_{A}-q_{A}\right)\left(j_{B}-q_{B}\right)+\sigma_{A A}\left(j_{B}-q_{B}\right)^{2}}{2\left(\sigma_{A A} \sigma_{B B}-\sigma_{A B}^{2}\right)}
\end{array}
$$

Note that instead of (29), one could write Langevin equations for the currents

$$
\begin{aligned}
& j_{A}=-\frac{d \rho_{A}}{d x}-\beta \rho_{A}\left(\rho_{B}-\rho_{C}\right)+\frac{\eta_{A}(x, \tau)}{\sqrt{L}} \\
& j_{B}=-\frac{d \rho_{B}}{d x}-\beta \rho_{B}\left(\rho_{C}-\rho_{A}\right)+\frac{\eta_{B}(x, \tau)}{\sqrt{L}}
\end{aligned}
$$

with the following correlations of the white noises $\eta_{A}(x, \tau)$ and $\eta_{B}(x, \tau)$

$$
\left\langle\eta_{A}(x, \tau) \eta_{B}\left(x, \tau^{\prime}\right)\right\rangle=\sigma_{A B}\left(\rho_{A}(x, \tau), \rho_{B}(x, \tau)\right) \delta\left(\tau-\tau^{\prime}\right)
$$

and similar expressions for $\left\langle\eta_{A}(x, \tau) \eta_{A}\left(x, \tau^{\prime}\right)\right\rangle$ and $\left\langle\eta_{B}(x, \tau) \eta_{B}\left(x, \tau^{\prime}\right)\right\rangle$.

As always, the currents and the densities are related by the conservation laws

$$
\frac{d \rho_{A}}{d \tau}=-\frac{d j_{A}}{d x} \quad ; \quad \frac{d \rho_{B}}{d \tau}=-\frac{d j_{B}}{d x} .
$$

For a perturbation of the form (which satisfies these conservation laws)

$$
\begin{array}{r}
\rho_{A}(x, \tau)=r_{A}+k\left(a_{1} \cos [k x+\omega \tau]+a_{2} \sin [k x+\omega \tau]\right) \\
\rho_{B}(x, \tau)=r_{B}+k\left(b_{1} \cos [k x+\omega \tau]+b_{2} \sin [k x+\omega \tau]\right) \\
j_{A}(x, \tau)=-\beta r_{A}\left(r_{B}-r_{C}\right)-\omega\left(a_{1} \cos [k x+\omega \tau]+a_{2} \sin [k x+\omega \tau]\right) \\
j_{B}(x, \tau)=-\beta r_{B}\left(r_{C}-r_{A}\right)-\omega\left(b_{1} \cos [k x+\omega \tau]+b_{2} \sin [k x+\omega \tau]\right)
\end{array}
$$

where

$$
k=2 n \pi \quad \omega=\frac{2 m \pi}{T}
$$


with $n \in N$ and $m \in Z$ one expands (28,29) to second order in $a_{1}, a_{2}, b_{1}, b_{2}$ and the probability (27) becomes a Gaussian which leads to the following expectations

$$
\begin{gathered}
\left\langle a_{1} a_{2}\right\rangle=\left\langle b_{1} b_{2}\right\rangle=0 \\
\left\langle a_{1}^{2}\right\rangle=\left\langle a_{2}^{2}\right\rangle=-\frac{4 r_{A}}{L T} \frac{1}{\Gamma_{k}}\left(\beta^{2} k^{2}\left[4\left(1-4 r_{A}\right) r_{B} r_{C}-\left(1-2 r_{A}\right)^{2}\left(1-r_{A}\right)\right]\right. \\
\left.+2 \beta k\left(1-2 r_{A}\right)\left(r_{C}-r_{B}\right) \omega-\left(1-r_{A}\right)\left(k^{4}+\omega^{2}\right)\right) \\
\left\langle a_{1} b_{1}\right\rangle=\left\langle a_{2} b_{2}\right\rangle=-\frac{4 r_{A} r_{B}}{L T} \frac{1}{\Gamma_{k}}\left(-\beta^{2} k^{2}\left[4 r_{A} r_{B}-1-2 r_{C}+8 r_{C}^{2}\right]\right. \\
\left.+2 \beta k\left(r_{B}-r_{A}\right) \omega+\left(k^{4}+\omega^{2}\right)\right) \\
\left\langle a_{1} b_{2}\right\rangle=-\left\langle a_{2} b_{1}\right\rangle=\frac{1}{L T} \frac{24 \beta k^{3} r_{A} r_{B} r_{C}}{\Gamma_{k}}
\end{gathered}
$$

where $\Delta$ is defined in (21) and

$$
\Gamma_{k}=\beta^{4} k^{4} \Delta^{2}-2 \beta^{2} k^{2} \Delta\left(k^{4}-\omega^{2}\right)+\left(k^{4}+\omega^{2}\right)^{2} .
$$

In one adds up the contributions of all perturbations of the form (30), one gets for the correlation functions

$$
\begin{gathered}
\left\langle\rho_{A}(x) \rho_{A}(y)\right\rangle_{c}=\sum_{k, \omega} k^{2}\left\langle a_{1}^{2}\right\rangle \cos [k(y-x)] \\
\left\langle\rho_{A}(x) \rho_{B}(y)\right\rangle_{c}=\sum_{k, \omega} k^{2}\left\langle b_{1}^{2}\right\rangle \cos [k(y-x)]+k^{2}\left\langle a_{1} b_{2}\right\rangle \sin [k(y-x)]
\end{gathered}
$$

In the long time limit the sums over the discrete frequencies (31) can be replaced by an integral and one gets

$$
\begin{gathered}
\left\langle\rho_{A}(x) \rho_{A}(y)\right\rangle_{c}= \\
\frac{1}{L} \sum_{n \geq 1} \frac{r_{A}\left[\beta^{2}\left(3 r_{A}\left(1-2 r_{A}\right)^{2}-\Delta\left(2-5 r_{A}\right)\right)+8 \pi^{2} n^{2}\left(1-r_{A}\right)\right]}{4 \pi^{2} n^{2}-\beta^{2} \Delta} \cos (2 \pi n(y-x)) \\
\left\langle\rho_{A}(x) \rho_{B}(y)\right\rangle_{c}=\frac{1}{L} \sum_{n \geq 1} \frac{\left.r_{A} r_{B}\left[2 \beta^{2}\left(\Delta-3 r_{C}+6 r_{C}^{2}\right)\right)-8 \pi^{2} n^{2}\right]}{4 \pi^{2} n^{2}-\beta^{2} \Delta} \cos (2 \pi n(y-x)) \\
+\frac{1}{L} \sum_{n \geq 1} \frac{12 \beta \pi n r_{A} r_{B} r_{C}}{4 \pi^{2} n^{2}-\beta^{2} \Delta} \sin (2 \pi n(y-x))
\end{gathered}
$$


In the particular case of equal densities, one recovers (9]10).

Using the identities

$$
\begin{gathered}
2 \sum_{n \geq 1} \cos [2 \pi n(x-y)]=-1+\delta(x-y) \\
\frac{\cos \left[\alpha\left(\frac{1}{2}-x\right)\right]}{\alpha \sin \frac{\alpha}{2}}=\frac{\cos (\alpha x)+\cos (\alpha(1-x))}{\alpha \sin \alpha}=\frac{2}{\alpha^{2}}-\sum_{n \geq 1} \frac{4}{4 \pi^{2} n^{2}-\alpha^{2}} \cos 2 n \pi x \\
\frac{\sin \left[\alpha\left(\frac{1}{2}-x\right)\right]}{\sin \frac{\alpha}{2}}=\frac{\cos (\alpha x)-\cos (\alpha(1-x))}{1-\cos \alpha}=\sum_{n \geq 1} \frac{8 n \pi}{4 \pi^{2} n^{2}-\alpha^{2}} \sin 2 n \pi x \\
\left(1-2 r_{A}\right)^{2}+\Delta=4 r_{B} r_{C}
\end{gathered}
$$

one can rewrite (33134) as

$$
\begin{aligned}
& \left\langle\rho_{A}(x) \rho_{A}(y)\right\rangle_{c}= \\
& \frac{1}{L}\left[r_{A}\left(1-r_{A}\right)(\delta(y-x)-1)-3 \beta r_{A}^{2} r_{B} r_{C}\left(\frac{\cos \left(\frac{\beta \sqrt{\Delta}}{2}(1-2 y+2 x)\right)}{\sqrt{\Delta} \sin \frac{\beta \sqrt{\Delta}}{2}}-\frac{2}{\beta \Delta}\right)\right]
\end{aligned}
$$

$$
\begin{aligned}
& \left\langle\rho_{A}(x) \rho_{B}(y)\right\rangle_{c}= \\
& \frac{1}{L}\left[-r_{A} r_{B}(\delta(y-x)-1)+\frac{3}{2} \beta r_{A} r_{B} r_{C}\left(1-2 r_{C}\right)\left(\frac{\cos \left(\beta \frac{\sqrt{\Delta}}{2}(1-2 y+2 x)\right)}{\sqrt{\Delta} \sin \frac{\beta \sqrt{\Delta}}{2}}-\frac{2}{\beta \Delta}\right)\right. \\
& \left.+\frac{3}{2} \beta r_{A} r_{B} r_{C} \frac{\sin \left(\frac{\beta \sqrt{\Delta}}{2}(1-2 y+2 x)\right)}{\sin \frac{\beta \sqrt{\Delta}}{2}}\right]
\end{aligned}
$$

which are totally equivalent to the expressions (23)|24).

As mentioned before, the large deviation function (66) of the density is only known (8) at order $\beta^{2}$ for the $A B C$ model. The knowledge (36,37) imposes constraints on the large deviation function. Trying to generalize (8), we found the following expression for the large deviation functional (6)

$$
\mathcal{F}\left[\rho_{A}(x), \rho_{B}(x), \rho_{C}(x)\right]=
$$




$$
\begin{aligned}
& \kappa+\int_{0}^{1} d x\left[\rho_{A}(x) \ln \rho_{A}(x)+\rho_{B}(x) \ln \rho_{B}(x)+\rho_{C}(x) \ln \rho_{C}(x)\right] \\
& -\beta \int_{0}^{1} d x \int_{0}^{1} d z \frac{\sin \left(c\left(\frac{1}{2}-z\right)\right)}{2 \sin \left(\frac{c}{2}\right)}\left[\rho_{A}(x) \rho_{B}(x+z)+\rho_{B}(x) \rho_{C}(x+z)+\rho_{C}(x) \rho_{A}(x+z)\right] \\
& -3 \beta^{2} \int_{0}^{1} d x \int_{0}^{1} d z \frac{\cos \left(c\left(\frac{1}{2}-z\right)\right)}{4 c \sin \left(\frac{c}{2}\right)}\left[r_{C}\left(1-3 r_{C}\right) \rho_{A}(x) \rho_{B}(x+z)\right. \\
& \left.\quad+r_{A}\left(1-3 r_{A}\right) \rho_{B}(x) \rho_{C}(x+z)+r_{B}\left(1-3 r_{B}\right) \rho_{C}(x) \rho_{A}(x+z)\right]
\end{aligned}
$$

where

$$
c=\beta \sqrt{\Delta-9 r_{A} r_{B} r_{C}}
$$

was compatible both with (8) at order $\beta^{2}$ for arbitrary density fluctuations and with the exact expressions of the correlation functions (36 37), i.e. for arbitrary $\beta$ but small deviations of the density from the flat profiles. What this expression becomes for general $\beta$ and arbitrary deviations of the density is an open question.

The macroscopic fluctuation theory developed by Bertini et al. [21, 22] (see also [26]) relates the steady state large deviation functional (6]) to the dynamical large deviation functional (27,28). One can try to apply this general procedure to the ABC model. We fix $0<\beta<\beta_{c}$ such that the steady state profiles are flat (equal to $r_{A}, r_{B}$ ) and such that the hydrodynamic equations (25) relax to $r_{A}, r_{B}$ for any initial density profiles (this property excludes situations with metastable profiles). Then the density large deviation functional (6) is given by

$$
\mathcal{F}\left[\rho_{A}(x), \rho_{B}(x), \rho_{C}(x)\right]=\inf _{\hat{\rho}, \hat{\jmath}}\left\{\mathcal{I}_{[-\infty, 0]}(\hat{\rho}, \hat{\jmath})\right\}
$$

where the infimum of the functional $\mathcal{I}_{[-\infty, 0]}(28)$ is taken over the trajectories $(\hat{\rho}(x, t), \hat{\mathrm{j}}(x, t))=\left\{\hat{\rho}_{A}(x, t), \hat{\rho}_{B}(x, t), \hat{\mathrm{j}}_{A}(x, t), \hat{\mathrm{j}}_{B}(x, t)\right\}$ starting at time $-\infty$ from the steady state configurations $\left(r_{A}, r_{B}\right)$ and ending at time $t=0$ at the configuration $\left(\rho_{A}(x), \rho_{B}(x)\right)$ (with $\left.\rho_{C}(x)=1-\rho_{A}(x)-\rho_{B}(x)\right)$. To recover the large deviation functional in the steady state, there is no other constraint on the currents $\hat{j}$ besides the conservation law $\partial_{t} \hat{\rho}=-\partial_{x} \hat{\jmath}$. Finding the optimal trajectory is in general an open problem which has been solved only in rare instances [22, 27]. For the sake of completeness, the equations satisfied by the optimal trajectories are presented in Appendix II. 
A perturbative analysis of the variational problem (39) leads to an expansion of $\mathcal{F}$ in the vicinity of the steady state. For example (38) can be obtained as the second order expansion of $\mathcal{F}$ and the two-point correlations can then be recovered by inverting the quadratic form in (38). Expanding $\mathcal{F}$ at the second order is essentially equivalent to the mode decomposition implemented in this section (30). To see this, we first note that (39) can be rewritten as a variational principle on the time interval $[-\infty, \infty]$ with the constraint that the trajectory $\hat{\rho}$ is equal to $\left(\rho_{A}(x), \rho_{B}(x)\right)$ at time 0

$$
\mathcal{F}\left[\rho_{A}(x), \rho_{B}(x), \rho_{C}(x)\right]=\inf _{\hat{\rho}, \hat{\jmath}}\left\{\mathcal{I}_{[-\infty, \infty]}(\hat{\rho}, \hat{\jmath})\right\}
$$

This identity comes from the fact that the contribution for positive times is null as one can choose $\hat{\rho}$ which relaxes to equilibrium according to (25). For small deviations, $\mathcal{I}_{[-\infty, \infty]}(\hat{\rho}, \hat{j})$ can then be expanded with respect to the Fourier modes (30). The different modes decouple and we recover a quadratic form with coefficients related to the correlations (32). The constraint on the density at time 0 is then achieved by optimizing this quadratic form over the temporal modes $\omega$. This leads to a second order expansion of $\mathcal{F}$ (in terms of the spatial Fourier modes) from which the steady state two-point correlation functions (33, 334) can be obtained.

\section{Diffusive systems on a ring}

The second example we analyze is the phase transition [29, 16, 28] which is expected to occur in the large deviation function of the current in some diffusive systems such as the weakly asymmetric exclusion process (WASEP).

The WASEP on a ring is defined as follows: one considers a fixed number $N=L r$ of particles on a ring of $L$ sites, with at most one particle per site. Each particle hops to its neighboring site on its right with rate $q^{-1}$ and to its neighboring site on its left with rate $q$, provided that the target site is empty. As for the ABC model, we consider here the diffusive regime where $q$ scales with the system size $L$ as in (3)

$$
q=\exp \left[-\frac{\beta}{L}\right] .
$$

For general diffusive systems with one species of particles on a ring, one can write an expression very similar to (27, 29) for the probability of observing a 
density profile $\rho(x, \tau)$ and a (rescaled) current $j(x, \tau)$ over a (rescaled) time interval $T$ (the microscopic time $t=T L^{2}$ )

$$
\operatorname{Pro}(\rho, j) \sim \exp \left(-L \mathcal{I}_{[0, T]}(\rho, j)\right)
$$

where

$$
\mathcal{I}_{[0, T]}(\rho, j)=\int_{0}^{1} d x \int_{0}^{T} d \tau \mathcal{H}(\rho, j)
$$

with

$$
\mathcal{H}(\rho, j)=\frac{\left[j(x, \tau)+D(\rho(x, \tau)) \frac{d \rho(x, \tau)}{d x}-\beta \sigma(\rho(x, \tau))\right]^{2}}{2 \sigma(\rho(x, \tau)} .
$$

and the current $j$ and the density $\rho$ are related, as usual, by the conservation law

$$
\frac{d \rho}{d \tau}=-\frac{d j}{d x}
$$

We refer to [28, 29] for further details on the derivation of (42). The functions $D(\rho)$ and $\sigma(\rho)$ in (43) are two functions characteristic of the diffusive system. For the WASEP, as defined above, these two functions are $D(\rho)=1$ and $\sigma(\rho)=2 \rho(1-\rho)$. In general their ratio is related to the compressibility at equilibrium by the Einstein relation (see [30] equation (4.6.1) or [31]).

If one considers the flux $Q_{t}(i)$ of particles between sites $i$ and $i+1$ during a long microscopic time $t$, the large deviation function $G\left(j_{0}\right)$ of the current describes the distribution of $Q_{t}(i)$, in the long time limit

$$
\operatorname{Pro}\left(\frac{Q_{t}(i)}{t}=\frac{j_{0}}{L}\right) \sim \exp \left[-\frac{t}{L} G\left(j_{0}\right)\right]
$$

(the $L$ dependence which appears in (45) can be understood because the system is diffusive [28, 29]). For systems with conservative dynamics such as the WASEP, which are irreducible Markov processes with a finite number of states, the large deviation function $G\left(j_{0}\right)$ depends neither on the initial condition at time 0 nor on the final configuration at time $t$ nor on the section $i$ chosen.

In what follows we consider (instead of the flux $Q_{t}(i)$ through a section) the total flux $Q_{t}$

$$
Q_{t}=\sum_{i=1}^{L} Q_{t}(i)
$$


which has the same large deviation function

$$
\operatorname{Pro}\left(\frac{Q_{t}}{t}=j_{0}\right) \sim \exp \left[-\frac{t}{L} G\left(j_{0}\right)\right] .
$$

According to the macroscopic fluctuation theory, one can calculate this large deviation function $G\left(j_{0}\right)$ by looking at the time evolution of the density and of the current which maximizes (41) with the constraint that

$$
\frac{1}{T} \int_{0}^{T} d \tau \int_{0}^{1} d x j(x, \tau)=j_{0}
$$

so that

$$
G\left(j_{0}\right)=\lim _{T \rightarrow \infty} \frac{1}{T}\left\{\min _{\rho(x, \tau), j(x, \tau)} \mathcal{I}_{[0, T]}(\rho, j)\right\}
$$

When the optimal density and current profiles in (49) are constant in time and in space [29], the expression of $G\left(j_{0}\right)$ follows immediately

$$
G\left(j_{0}\right)=\frac{\left[j_{0}-\beta \sigma(r)\right]^{2}}{2 \sigma(r)}
$$

where $r=N / L$ is the density of particles along the ring.

By analyzing the neighborhood of the flat profile solution, it was shown in [16] that this profile is locally unstable when

$$
8 \pi^{2} D^{2}(r) \sigma(r)+\left[\beta^{2} \sigma^{2}(r)-j_{0}^{2}\right] \sigma^{\prime \prime}(r)<0
$$

and the optimal profiles becomes space or space and time dependent. (Note that, as for the $A B C$ model, (51) is obtained by a local stability analysis. Thus the optimal profiles might already be space or space and time dependent even when (51) is not satisfied with the occurrence of first order transitions).

We are now going to see that, although there is no trace of the second order phase transition (51) in the expression (50), the correlation functions become singular along the stability line (51). There are several ways of defining the correlation functions, conditioned on the current: if the flux is conditioned to be $Q_{t}=j_{0} t$ over a time interval $-t / 2<t^{\prime}<t / 2$, one expects the correlation $\left\langle\rho\left(x, t^{\prime}\right) \rho\left(y, t^{\prime}\right)\right\rangle_{c}$ to depend on whether $t^{\prime}$ is close or far from the boundaries of the time interval $(-t / 2, t / 2)$. We are now going to calculate two of these correlation functions

$$
\langle\rho(x) \rho(y)\rangle_{\text {intermediate }}=\lim _{t \rightarrow \infty}\left\langle\rho(x, 0) \rho(y, 0) \mid Q_{t}=j_{0} t\right\rangle_{c}
$$




$$
\langle\rho(x) \rho(y)\rangle_{\text {final }}=\lim _{t \rightarrow \infty}\left\langle\rho(x, t / 2) \rho(y, t / 2) \mid Q_{t}=j_{0} t\right\rangle_{c}
$$

During the macroscopic time interval $[-T / 2, T / 2]$, one considers a small perturbation of the flat profile $r$ of the form

$$
\begin{array}{r}
\rho(x, \tau)=r+k\left[f_{1}(\tau) \cos (k x)+f_{2}(\tau) \sin (k x)\right] \\
j(x, \tau)=I_{0}(\tau)+f_{2}^{\prime}(\tau) \cos (k x)-f_{1}^{\prime}(\tau) \sin (k x)
\end{array}
$$

where $f_{1}(\tau)$ and $f_{2}(\tau)$ are a priori two arbitrary functions and $k=2 \pi n$ with $n \geq 1$, integer). The parameter $I_{0}(\tau)$ takes into account the constraint on the current in the time interval $[-T / 2, T / 2]$

$$
\left\{\begin{array}{l}
I_{0}(\tau)=j_{0}, \quad \text { if }-T / 2<\tau<T / 2, \\
I_{0}(\tau)=\beta \sigma(r), \quad \text { otherwise }
\end{array}\right.
$$

One can rewrite (43) to quadratic order

$$
\mathcal{H}(\rho, j)=A(\tau)\left(f_{1}^{2}+f_{2}^{2}\right)+B(\tau)\left(f_{1}^{\prime 2}+{f_{2}^{\prime}}^{2}\right)+C(\tau)\left(f_{1} f_{2}^{\prime}-f_{2} f_{1}^{\prime}\right)+E(\tau)\left(f_{1} f_{1}^{\prime}+f_{2} f_{2}^{\prime}\right)
$$

where

$$
\begin{aligned}
& A(\tau)=\frac{D^{2} k^{4}}{4 \sigma}+\frac{\beta^{2} k^{2} \sigma^{\prime \prime}}{8}+\frac{k^{2} \sigma^{\prime 2} I_{0}(\tau)^{2}}{4 \sigma^{3}}-\frac{k^{2} \sigma^{\prime \prime} I_{0}(\tau)^{2}}{8 \sigma^{2}} \\
& B(\tau)=\frac{1}{4 \sigma}, \quad C(\tau)=-\frac{k \sigma^{\prime} I_{0}(\tau)}{2 \sigma^{2}}, \quad E(\tau)=\frac{D k^{2}}{2 \sigma}
\end{aligned}
$$

with the functions $D, \sigma, \sigma^{\prime}, \sigma^{\prime \prime}$ evaluated at $\rho=r=N / L$.

When $T$ goes to infinity, the expectations $\left\langle f_{1}^{2}(0)\right\rangle,\left\langle f_{1}(0) f_{1}(0)\right\rangle,\left\langle f_{2}^{2}(0)\right\rangle$ can then be computed in the following two situations (see Appendix I):

- in the case (52), one has $I_{0}(\tau)=j_{0}$ for all times $\tau$. One gets from (64)

$$
\left\langle f_{1}(0)^{2}\right\rangle=\left\langle f_{2}(0)^{2}\right\rangle=\frac{\sigma}{L \sqrt{D^{2} k^{4}+\frac{\beta^{2} k^{2} \sigma \sigma^{\prime \prime}}{2}-\frac{k^{2} \sigma^{\prime \prime} j_{0}^{2}}{2 \sigma}}}
$$

and by summing the contributions of all the modes, this gives

$$
\langle\rho(x) \rho(y)\rangle_{\text {intermediate }}=\frac{2 \pi}{L} \sum_{n \geq 1} \frac{n \cos (2 \pi n(y-x)) \sigma}{\sqrt{4 \pi^{2} n^{2} D^{2}+\frac{\beta^{2} \sigma \sigma^{\prime \prime}}{2}-\frac{\sigma^{\prime \prime} j_{0}^{2}}{2 \sigma}}} .
$$


- in the case (53), one has $I_{0}(\tau)=j_{0}$ for $\tau \leq 0$ and $I_{0}(\tau)=\beta \sigma(r)$ for $\tau>0$

$$
\left\langle f_{1}(0)^{2}\right\rangle=\left\langle f_{2}(0)^{2}\right\rangle=\frac{2 \sigma}{L\left[D k^{2}+\sqrt{D^{2} k^{4}+\frac{\beta^{2} k^{2} \sigma \sigma^{\prime \prime}}{2}-\frac{k^{2} \sigma^{\prime \prime} j_{0}^{2}}{2 \sigma}}\right]}
$$

and the sum over all the modes leads to

$$
\langle\rho(x) \rho(y)\rangle_{\text {final }}=\frac{4 \pi}{L} \sum_{n \geq 1} \frac{n \cos (2 \pi n(y-x)) \sigma}{2 \pi n D+\sqrt{4 \pi^{2} n^{2} D^{2}+\frac{\beta^{2} \sigma \sigma^{\prime \prime}}{2}-\frac{\sigma^{\prime \prime} j_{0}^{2}}{2 \sigma}}} .
$$

We see in expressions (58,60) that the correlation functions become singular along the transition line (51), where the $n=1$ mode becomes unstable. Note that only the intermediate correlation function (58) diverges at the transition.

Let $\bar{j}_{0}=\beta \sigma(r)$ be the mean current, then one can rewrite the correlation function (58) as

$$
\begin{aligned}
& \langle\rho(x) \rho(y)\rangle_{\text {intermediate }}= \\
& \quad \frac{\sigma}{2 L D} \sum_{n \geq 1} 2 \cos (2 \pi n(y-x))\left(1+\left[\frac{1}{\sqrt{1-\frac{\sigma^{\prime \prime}}{8 \sigma \pi^{2} n^{2} D^{2}}\left(j_{0}^{2}-\left(\bar{j}_{0}\right)^{2}\right)}}-1\right]\right) .
\end{aligned}
$$

When there is no constraint on the current then $j_{0}=\bar{j}_{0}$ and (61) reduces (see (35)) to

$\langle\rho(x) \rho(y)\rangle_{\text {intermediate }}=\frac{\sigma}{2 L D} \sum_{n \geq 1} 2 \cos (2 \pi n(y-x))=\frac{\sigma}{2 L D}(\delta(x-y)-1)$,

which are the correlations for the (micro-canonical) invariant measure of the diffusive part of the dynamics. This was already emphasized in [6, 25]. On the other hand, if the system is conditioned to an atypical current deviation, the term in the bracket in (61) does not vanish. It is interesting to note that all the modes have the same sign which depends on $j_{0}^{2}-\bar{j}_{0}^{2}$ and $\sigma^{\prime \prime}$. One can check that depending on the sign of $j_{0}^{2}-\bar{j}_{0}^{2}$ the correlations for $x-y$ small are either positive or negative: the particles tend to cluster or to spread according to the constraint on the current. We interpret this clustering phenomenon as a precursor of the macroscopic clustering which occurs after the transition.

Instead of the perturbation (54), one could also have used a space/time decomposition over the Fourier modes as in (30). However this would have 
led to extra difficulties to treat the fluctuations at the final time. In the latter case, the functional depends on the current constraint which is not uniform in time so that the contributions of the modes at different frequencies would be coupled (unlike the case of intermediate fluctuations). In the particular case of the WASEP, a microscopic approach based on a truncation procedure as in section 2.2 would also have led to the correlations (58).

In [19], it was shown recently that a very similar phase transition occurs in the SSEP (the symmetric exclusion process) when one considers the large deviation function of the activity $K_{t}$ (which is the total number of changes

of configurations during time $t$ ). A calculation almost identical to the one presented in this section can be done to calculate the correlation functions, conditioned on the value of $K_{t}$. The nature of the singularities of the correlation functions are then very similar to those found in (58, 60).

\section{Conclusion}

In the present work, we have calculated, using two different approaches, the long range correlation functions in the $A B C$ model and shown that they become singular at the second order phase transition (9, 10,33,34,36/37). We have also calculated, for general diffusive systems, the two point correlation functions, conditioned on the current (58, 60) and seen that they become singular at a second order phase transition. Similar calculations can be done, when conditioned on other quantities such as the activity [19].

It would be interesting to try to extend our results to other phases than the flat phase or to other situations than the ring geometry, like open systems. The large scale Gaussian fluctuations of the density, which here are at the origin of the long range correlations, allow also to calculate all the cumulants of the current [19] for diffusive systems. It would be interesting to see whether one could establish more direct or more general relations between the long range correlations and the distribution of the current, and to know how these relations are modified in the case of open systems.

Lastly we have obtained an improved expression (38) of the large deviation functional of the density for the $A B C$ model. This expression is still an approximation. It would be interesting to go further, for example by calculating higher correlations using the truncation procedure (16]17). 
Acknowledgments. TB and BD acknowledge the support of the French Ministry of Education through the ANR BLAN07-2184264 grant. FvW acknowledges the support of the French Ministry of Education through the ANR-05JCJC-44482 grant.

\section{Appendix I}

In this appendix, we establish the expressions (5759). Let us consider two Gaussian functions $h_{1}(\tau), h_{2}(\tau)$ of the time $\tau$ distributed according to the following distribution

$$
\begin{gathered}
\operatorname{Pro}\left(\left\{h_{1}(\tau), h_{2}(\tau)\right\}\right) \sim \exp \left[-L \int_{-\infty}^{\infty} d \tau\left[A(\tau)\left(h_{1}^{2}+h_{2}^{2}\right)+B(\tau)\left(h_{1}^{\prime 2}+h_{2}^{\prime 2}\right)\right.\right. \\
\left.+C(\tau)\left(h_{1} h_{2}^{\prime}-h_{1}^{\prime} h_{2}\right)+E(\tau)\left(h_{1} h_{1}^{\prime}+h_{2} h_{2}^{\prime}\right)\right]
\end{gathered}
$$

Here we are interested in situations where the functions $A(\tau), B(\tau), C(\tau), E(\tau)$ take some constant values $A_{-}, B_{-}, C_{-}, E_{-}$for $\tau<0$ and other constant values (possibly the same) $A_{+}, B_{+}, C_{+}, E_{+}$for $\tau>0$.

If we fix $h_{1}(0)$ and $h_{2}(0)$, and one integrates (62) over $h_{1}(\tau)$ and $h_{2}(\tau)$ for all times $\tau \neq 0$ one gets

$\operatorname{Pro}\left(\left\{h_{1}(0), h_{2}(0)\right\}\right) \sim \exp \left[-L\left(\alpha_{+} B_{+}+\alpha_{-} B_{-}+\frac{E_{-}-E_{+}}{2}\right)\left(h_{1}(0)^{2}+h_{2}(0)^{2}\right)\right]$

where

$$
\alpha_{+}=\frac{\sqrt{4 A_{+} B_{+}-C_{+}^{2}}}{2 B_{+}} \quad ; \quad \alpha_{-}=\frac{\sqrt{4 A_{-} B_{-}-C_{-}^{2}}}{2 B_{-}}
$$

In fact it can be shown that the functions which maximize (62) for $\tau>0$ are, at fixed $h_{1}(0), h_{2}(0)$

$$
\begin{aligned}
& h_{1}(\tau)=e^{-\alpha_{+} \tau}\left[h_{1}(0) \cos \left(\beta_{+} \tau\right)+h_{2}(0) \sin \left(\beta_{+} \tau\right)\right] \\
& h_{2}(\tau)=e^{-\alpha_{+} \tau}\left[h_{2}(0) \cos \left(\beta_{+} \tau\right)-h_{1}(0) \sin \left(\beta_{+} \tau\right)\right]
\end{aligned}
$$

where

$$
\beta_{+}=\frac{C_{+}}{2 B_{+}} .
$$

Similar expressions with $\alpha_{+}$and $\beta_{+}$replaced by $-\alpha_{-}$and $\beta_{-}$give these optimal functions for $\tau<0$. This leads to

$$
\left\langle h_{1}(0)^{2}\right\rangle=\left\langle h_{2}(0)^{2}\right\rangle=\frac{1}{\left[2\left(\alpha_{+} B_{+}+\alpha_{-} B_{-}\right)+E_{-}-E_{+}\right] L}
$$

and $\left\langle h_{1}(0) h_{2}(0)\right\rangle=0$. 


\section{Appendix II}

In this Appendix, we study the variational problem (39). We set

$\Sigma(\hat{\rho})=\left(\begin{array}{cc}2 \rho_{A}\left(1-\rho_{A}\right) & -2 \rho_{A} \rho_{B} \\ -2 \rho_{A} \rho_{B} & 2 \rho_{B}\left(1-\rho_{B}\right)\end{array}\right) \quad F(\hat{\rho})=\left(\begin{array}{c}\rho_{A}\left(\rho_{A}+2 \rho_{B}-1\right) \\ \rho_{B}\left(1-2 \rho_{A}-\rho_{B}\right)\end{array}\right)$

The trajectories are denoted by $\hat{\rho}=\left(\rho_{A}, \rho_{B}\right)$ and the currents $\hat{\jmath}=\left(j_{A}, j_{B}\right)$. It will be convenient to rewrite the currents in terms of the new variables $H=\left(H_{A}, H_{B}\right)$

$$
\hat{\jmath}(x, t)=-\partial_{x} \hat{\rho}(x, t)-\beta F(\hat{\rho}(x, t))+\Sigma(\hat{\rho}(x, t)) \partial_{x} H(x, t),
$$

with $H(0, t)=H(1, t)=0$. In particular

$$
\partial_{t} \hat{\rho}(x, t)=\partial_{x}^{2} \hat{\rho}(x, t)+\beta \partial_{x}\left(F(\hat{\rho}(x, t))-\partial_{x}\left(\boldsymbol{\Sigma}(\hat{\rho}(x, t)) \partial_{x} H(x, t)\right)\right) .
$$

Thus the functional (28) reads

$$
\mathcal{I}_{[-\infty, 0]}(\hat{\rho}, \hat{\mathrm{j}})=\frac{1}{2} \int_{-\infty}^{0} d t \int_{0}^{1} d x\left(H_{A}^{\prime}, H_{B}^{\prime}\right) \Sigma(\rho(x, t))\left(\begin{array}{c}
H_{A}^{\prime} \\
H_{B}^{\prime}
\end{array}\right),
$$

where $H_{A}^{\prime}, H_{B}^{\prime}$ stand for the spatial derivatives. Optimizing the functional implies that the optimal trajectories satisfy

$$
\begin{aligned}
\partial_{t} \rho_{A} & =\Delta \rho_{A}+\beta \partial_{x}\left(\rho_{A}\left(\rho_{A}+2 \rho_{B}-1\right)\right)-2 \partial_{x}\left(\rho_{A}\left(1-\rho_{A}\right) H_{A}^{\prime}-\rho_{A} \rho_{B} H_{B}^{\prime}\right) \\
\partial_{t} \rho_{B} & =\Delta \rho_{B}+\beta \partial_{x}\left(\rho_{B}\left(1-2 \rho_{A}-\rho_{B}\right)\right)-2 \partial_{x}\left(\rho_{B}\left(1-\rho_{B}\right) H_{B}^{\prime}-\rho_{A} \rho_{B} H_{A}^{\prime}\right) \\
\partial_{t} H_{A} & =-\Delta H_{A}+\beta\left(2 \rho_{A}+2 \rho_{B}-1\right) H_{A}^{\prime}-2 \beta \rho_{B} H_{B}^{\prime}-\left(\left(1-2 \rho_{A}\right)\left(H_{A}^{\prime}\right)^{2}-2 \rho_{B} H_{A}^{\prime} H_{B}^{\prime}\right) \\
\partial_{t} H_{B} & =-\Delta H_{B}+2 \beta \rho_{A} H_{A}^{\prime}-\beta\left(2 \rho_{B}+2 \rho_{A}-1\right) H_{B}^{\prime}-\left(\left(1-2 \rho_{B}\right)\left(H_{B}^{\prime}\right)^{2}-2 \rho_{A} H_{A}^{\prime} H_{B}^{\prime}\right)
\end{aligned}
$$

with the constraint on the densities at time $0,\left(\rho_{A}(x, 0), \rho_{B}(x, 0)\right)=\left(\rho_{A}(x), \rho_{B}(x)\right)$ and $\left(r_{A}, r_{B}\right)$ at time $-\infty$.

Solving (67) amounts to knowing the functional $\mathcal{F}$ (see [22] equation $(2.16))$

$$
H_{A}=\frac{\partial \mathcal{F}}{\partial \rho_{A}}, \quad H_{B}=\frac{\partial \mathcal{F}}{\partial \rho_{B}}
$$


In the case $r_{A}=r_{B}=1 / 3$, the optimal drifts can be guessed from the explicit expression (17) of the functional $\mathcal{F}$

$$
\begin{aligned}
H_{A}(x)= & \log \left(\frac{\rho_{A}(x)}{1-\rho_{A}(x)-\rho_{B}(x)}\right)+\beta\left(\int_{0}^{x} d u \rho_{B}(u)-\rho_{A}(u)+\right. \\
& \left.\int_{x}^{1} d u\left(1-\rho_{B}(u)-\rho_{A}(u)\right)+\int_{0}^{1} d u(u-x)\left(3 \rho_{B}(u)-1\right)\right)
\end{aligned}
$$

and

$$
H_{A}^{\prime}(x)=\frac{\left(\rho_{B}(x)-1\right) \rho_{A}^{\prime}(x)-\rho_{A}(x) \rho_{B}^{\prime}(x)}{\rho_{A}(x)\left(\rho_{A}(x)+\rho_{B}(x)-1\right)}+\beta\left(3 \rho_{B}(x)-1\right) .
$$

A similar expression holds for $H_{B}$ leading to

$$
\begin{aligned}
& \partial_{t} \rho_{A}=-\Delta \rho_{A}(x, t)-\beta \partial_{x}\left(\rho_{A}\left(\rho_{A}+2 \rho_{B}-1\right)\right), \\
& \partial_{t} \rho_{B}=-\Delta \rho_{B}(x, t)-\beta \partial_{x}\left(\rho_{B}\left(1-2 \rho_{A}-\rho_{B}\right)\right) .
\end{aligned}
$$

Thus the optimal trajectories are the time reversed of the hydrodynamic equations (25). This could have been guessed from the microscopic reversibility derived in [14]. Under the assumption that the hydrodynamic equations (25) relax to the flat densities $r_{A}=r_{B}=1 / 3$, the expression (77) of $\mathcal{F}$ can be recovered from the optimal trajectories by integrating (66) over the time.

\section{References}

[1] H. Spohn, Long range correlations for stochastic lattice gases in a nonequilibrium steady state, J. Phys. A 16 4275-4291 (1983)

[2] R. Schmitz, E.G.D. Cohen, Fluctuations in a fluid under a stationary heat-flux .1. General theory J. Stat. Phys. 39 285-316 (1985)

[3] J.R. Dorfman, T.R. Kirkpatrick, J.V. Sengers, Generic long-range correlations in molecular fluids Annual Review of Physical Chemistry 45 213-239 (1994)

[4] J.M. Ortiz de Zarate, J.V. Sengers, On the physical origin of long-ranged fluctuations in fluids in thermal nonequilibrium states J. Stat. Phys. 115 1341-1359 (2004) 
[5] B. Derrida, C. Enaud, C. Landim, S. Olla, Fluctuations in the weakly asymmetric exclusion process with open boundary conditions, J. Stat. Phys. 118, 795-811 (2005)

[6] L. Bertini, A. De Sole, D. Gabrielli, G. Jona-Lasinio, C. Landim, On the long range correlations of thermodynamic systems out of equilibrium cond-mat arXiv:0705.2996, (2007)

[7] B. Derrida, J.L. Lebowitz, E.R. Speer, Entropy of open lattice systems, J. Stat. Phys. 126 1083-1108 (2007)

[8] B. Derrida, Non-equilibrium steady states: fluctuations and large deviations of the density and of the current J. Stat. Mech. P07023 (2007)

[9] M.R. Evans, Phase transitions in one-dimensional nonequilibrium systems, Braz. J. Phys. 30, 42-57 (2000)

[10] Y. Kafri, E. Levine, D. Mukamel, G.M. Schütz, J. Torok, Criterion for phase separation in one-dimensional driven systems, Phys. Rev. Lett. 89, $035702(2002)$

[11] A. Parmeggiani, T. Franosch, E. Frey, Phase coexistence in driven onedimensional transport Phys. Rev. Lett. 90086601 (2003)

[12] M.R. Evans, Y. Kafri, H.M. Koduvely, D. Mukamel, Phase separation and coarsening in one-dimensional driven diffusive systems Phys. Rev. E 58 2764-2778 (1998)

[13] M.R. Evans, Y. Kafri, H.M. Koduvely, D. Mukamel, Phase separation in one-dimensional driven diffusive systems: Local dynamics leading to long-range Hamiltonians Phys. Rev. Lett. 80 425-429 (1998)

[14] M. Clincy, B. Derrida, M.R. Evans, Phase transitions in the ABC model, Phys. Rev. E 67, 066115 (2003)

[15] G. Fayolle, C. Furtlehner, Dynamical windings of random walks and exclusion models. Part I: Thermodynamic limit in Z(2), J. Stat. Phys. 114 229-260 (2004)

[16] T. Bodineau, B. Derrida, Distribution of current in nonequilibrium diffusive systems and phase transitions, Phys. Rev. E 72066110 (2005) 
[17] T. Bodineau, B. Derrida, Current fluctuations in non-equilibrium diffusive systems: an additivity principle Phys. Rev. Lett. 92, 180601, (2004)

[18] G. Fayolle, C. Furtlehner, Stochastic Dynamics of Discrete Curves and Multi-Type Exclusion Processes, J. Stat. Phys. 127 1049-1094 (2007)

[19] C. Appert-Rolland, B. Derrida, V. Lecomte, F. van Wijland, Universal cumulants of the current in diffusive systems on a ring, cond-mat arXiv:0804.2590, (2008)

[20] G.M. Schütz Critical phenomena and universal dynamics in onedimensional driven diffusive systems with two species of particles J. Phys. A: Math. Gen. 36 R339-R379 (2003)

[21] L. Bertini, A. De Sole, D. Gabrielli, G. Jona-Lasinio, C. Landim, Fluctuations in stationary non equilibrium states of irreversible processes, Phys. Rev. Lett. 87040601 (2001)

[22] L. Bertini, A. De Sole, D. Gabrielli, G. Jona-Lasinio, C. Landim, Macroscopic fluctuation theory for stationary non equilibrium states, J. Stat, Phys. 107, 635-675 (2002)

[23] L. Bertini, A. De Sole, D. Gabrielli, G. Jona-Lasinio, C. Landim, Large deviations for the boundary driven symmetric simple exclusion process, Math. Phys. Analysis and Geometry 6, 231-267 (2003)

[24] L. Bertini, A. De Sole, D. Gabrielli, G. Jona-Lasinio, C. Landim, Minimum dissipation principle in stationary non equilibrium states, J. Stat. Phys. 116 831-841 (2004)

[25] L. Bertini, A. De Sole, D. Gabrielli, G. Jona Lasinio, C. Landim, Stochastic interacting particle systems out of equilibrium, J. Stat. Mech. P07014. (2007)

[26] M. Freidlin, A. Wentzell, Random perturbations of dynamical systems. Springer-Verlag, (1998)

[27] L. Bertini, D. Gabrielli, J. Lebowitz, Large deviations for a stochastic model of heat flow, J. Stat. Phys. 121, no. 5-6, 843-885 (2005)

[28] T. Bodineau, B. Derrida, Cumulants and large deviations of the current in non-equilibrium steady states C.R. Physique 8 540-555 (2007) 
[29] L. Bertini, A. De Sole, D. Gabrielli, G. Jona-Lasinio, C. Landim, Non equilibrium current fluctuations in stochastic lattice gases J. Stat. Phys. 123 237-276 (2006)

[30] R. Kubo, M. Toda, N. Hashitsume, Statistical physics II. Nonequilibrium statistical mechanics, Springer Series in Solid-State Sciences. 31 (1998)

[31] H. Spohn, Large scale dynamics of interacting particles, Springer-Verlag, Berlin (1991)

[32] C. Kipnis, S. Olla, S. Varadhan, Hydrodynamics and large deviations for simple exclusion processes, Commun. Pure Appl. Math. 42, 115-137 (1989) 